\title{
Mononucleate da sangue periferico autologhe da filtrazione selettiva per il trattamento delle lesioni croniche dell'arto inferiore: Risultati a 4 anni
}

\author{
Matteo Bucalossi, ${ }^{1,2,3}$ Fabrizio Mariani ${ }^{1,2,3}$ \\ ${ }^{1}$ The Compression Therapy Study Group (CTG), Firenze; ${ }^{2}$ Unità Operativa Chirurgia, Valdisieve Hospital, Pontassieve (FI); ${ }^{3}$ European \\ Training Centre in Phlebology-European Union of Medical Specialists UEMS, Angiomedica Vein Clinic, Colle Val d'Elsa-Siena, Italia
}

\section{RIASSUNTO}

Recentemente è stato introdotto sul mercato una tecnologia a filtrazione selettiva per produrre un concentrato autologo di cellule mononucleate da sangue periferico (Peripheral Blood Mononuclear Cells, PBMNC) con indicazione d'uso per terapia cellulare umana. Le PBMNC trovano indicazione nel trattamento delle lesioni croniche degli arti inferiori. Lo scopo di questo studio è quello di valutare l'efficacia e la tollerabilità di tale trattamento in un gruppo di pazienti che presentavano ulcere cutanee degli arti inferiori, ad etiologia varia, non rispondono alle terapie topiche tradizionali ed avanzate. Lo studio è prospettico standardizzato. Sono state trattati 8 pazienti che presentavano in totale 22 ulcere cutanee ad etiologia variabile. Le PBMNC autologhe sono state impiantate lungo il decorso delle arterie tibiali di riferimento e nell'area peri-lesionale. Tutti i pazienti sono stati sottoposti ad un ciclo di tre infiltrazioni, eseguite in sala operatoria, con cadenza mensile, tranne un paziente che è stato sottoposto ad un quarto impianto, perché l'operatore ha ritenuto che uno ulteriore avrebbe accelerato lo stato già rigenerativo della cute stessa. Delle 22 ulcere trattate con tale metodologia, 14 sono giunte a guarigione completa entro un mese dalla fine dei tre impianti, mentre 8 sono andate verso un netto miglioramento. Tutti i pazienti hanno mostrato una buona compliance al trattamento e non sono stati registrati effetti avversi minori o maggiori. Il 50\% dei pazienti trattati è stato sottoposto a follow-up a quattro anni dalla fine del trattamento, e solo una paziente ha avuto un'ulcera cutanea recidiva, ma in altra sede da quella iniziale. Le PBMNC, prodotte con sistema a filtrazione selettiva, si sono dimostrate un trattamento efficace delle lesioni croniche a diversa eziologia degli arti inferiori. La guarigione di tutte le lesioni cutanee trattate in tempi rapidi, la netta riduzione del dolore, e l'assenza di recidive a quattro anni suggeriscono un effetto clinico duraturo nel tempo.

Corrispondenza: Matteo Bucalossi, The Compression Therapy Study Group (CTG), Via Kyoto 51, 50126 Firenze, Italia. Tel.: +39.3386892045.

E-mail: matteobucalossi@tiscali.it

Parole chiave: Mononucleate da sangue periferico (PBMNC); lesioni croniche arti inferiori; filtrazione selettiva; recidiva.

Conflitto di interessi: Gli autori dichiarano l'assenza di conflitto di interessi.

Disponibilità di dati e materiali: Tutti i dati analizzati in questo studio sono presenti nel seguente articolo.

Approvazione etica e consenso alla partecipazione: Questo studio è conforme a quanto stabilito dalla dichiarazione di Helsinki del 1964 , riveduta nel 2013. I pazienti coinvolti in questo studio hanno fornito il loro consenso alla partecipazione.

Consenso alla pubblicazione: I pazienti hanno fornito il loro consenso alla pubblicazione dei dati e delle immagini presenti in questo articolo.

Ricevuto per la pubblicazione: 13 ottobre 2020 .

Accettato per la pubblicazione: 30 marzo 2021.

This work is licensed under a Creative Commons Attribution NonCommercial 4.0 License (CC BY-NC 4.0).

${ }^{\circ}$ Copyright: the Author(s), 2021

Licensee PAGEPress, Italy

Italian Journal of Wound Care 2021; 5(2):69

doi:10.4081/ijwc.2021.69

\section{INTRODUZIONE}

Le ulcere cutanee degli arti inferiori rappresentano l'esito di diversi processi patologici e costituiscono una patologia sempre più attuale, sia per la loro frequenza che per le problematiche terapeutiche che esse comportano.

Colpiscono circa $1{ }^{\prime} 1 \%$ della popolazione adulta dei paesi sviluppati (con oltre il 3\% degli ultrasettantenni) e proprio per le sue caratteristiche è da considerarsi una vera e propria piaga sociale, coinvolgendo, oltre il diretto interessato, anche tutto l'ambiente familiare circostante. ${ }^{1}$

Inoltre, le cifre degli studi sono spesso sottostimate, infatti non è infrequente che il primo approccio terapeutico viene effettuato dal paziente stesso, sfruttando esperienze di amici o vicini di casa. In caso di mancato risultato o peggioramento del quadro clinico, viene interpellato il medico di medicina generale e, solo in terza istanza, il paziente viene inviato in centri specializzati in vulnologia. ${ }^{2}$

Le terapie tutt'oggi proposte per la cura delle ulcere cutanee sono state molte e spesso indaginose, ma con caratteristica comune quella di dare risultati alterni o addirittura insoddisfacenti.

La terapia cellulare autologa rappresenta un approccio innovativo e promettente per la rigenerazione dei tessuti lesionati, in particolare nuove evidenze scientifiche indicano le mononucleate totali da sangue periferico, costituite dalle popolazioni di monociti/macrofagi e linfociti, come cellule 
ad alta capacità angiogenica e arteriogenica $\mathrm{a}^{3-5} \mathrm{e}$ vasculogenica ed in generale fondamentali nei processi di rigenerazione dei tessuti stessi. ${ }^{6-8}$

Le cellule mononucleate da sangue periferico, indicate nella letteratura scientifica come PB-MNC (Peripheral Blood Mononuclear Cells), rappresentano un concentrato cellulare autologo per il trattamento di pazienti con ischemia critica con ulcere cutanee complesse degli arti inferiori., ${ }^{910}$

Recentemente una meta-analisi ha indicato le PBMNC come il solo concentrato cellulare autologo in grado di ridurre significativamente le amputazioni nei pazienti con ischemia critica non-option. ${ }^{11}$ La nostra osservazione include ulcere croniche che colpiscono gli arti inferiori, infatti queste lesioni sono frequentemente resistenti alle normali terapie per numerosi fattori, tra i quali: i) i disturbi circolatori, venosi o arteriosi che siano, che colpiscono preferenzialmente gli arti inferiori; ii) la gamba è una delle sedi più esposte all'azione dei traumi, infatti questi, su di un arto già compromesso da insufficienza circolatoria, possono provocare lo sviluppo di lesioni cutanee ad andamento torpido; iii) il piede ed il terzo inferiore di gamba vengono ispezionati o puliti meno frequentemente rispetto ad altre parti del corpo e per cui sono più facilmente sede di infezioni e, a loro volta, causa di lesioni cutanee croniche; iv) l'incidenza della malattia diabetica, associata a micro- e macro-angiopatia degli arti inferiori, è in notevole aumento tra la popolazione dei paesi sviluppati.

Lo scopo di questo studio è quello di riportare ed analizzare l'esperienza maturata, presso il centro vulnologico specializzato della Valdisieve Hospital, nell'utilizzo della terapia cellulare con PBMNC prodotte da sistema a filtrazione selettiva, con indicazione d'uso per terapia cellulare umana, in un gruppo di pazienti che presentavano ulcere croniche agli arti inferiori, ad etiologia varia, resistenti alle comuni terapie topiche, medicazioni avanzate comprese, ed inoltre classificate come "difficili", cioè presenti da almeno sei mesi in maniera continuativa.

\section{MATERIALI E METODI}

\section{Pazienti}

L'esperienza clinica con le PBMNC è stata da noi condotta su un gruppo di pazienti che presentavano ulcerazione cronica degli arti inferiori, ad etiologia varia, refrattaria ad ogni terapia topica e presente da più di sei mesi.

Il protocollo prevedeva un'anamnesi accurata del paziente, un'attenta visita specialistica con esame ecocolor-doppler artero-venoso, e misurazione, con fotografia dell'ulcera, in modo da classificare dettagliatamente sia l'etiologia che le dimensioni iniziali della lesione.

Dall'ottobre 2013 a gennaio 2015, sono state trattate 22 ulcere cutanee, ad etiologia varia, di 8 pazienti, 4 di sesso maschile 4 di sesso femminile, con un'età media di circa 86 anni (range 73-94 anni). Tutte le lesioni erano presenti da almeno sei mesi in maniera continuativa e non avevano riposto ai trattamenti standard.

Le ulcere cutanee reclutate all'interno dello studio si dividono in 12 di tipo arterioso, 3 miste, 6 di tipo dismetabolico su base diabetica, ed 1 di tipo iatrogeno, in conseguenza ad un intervento di ricostruzione del tendine calcaneale (Tabella 1).

Le dimensioni delle ulcere cutanee sono state quanto mai disomogenee, infatti la superficie delle lesioni cutanee variava da 5 a $168 \mathrm{~cm}^{2}$; anche perché le ulcere con dimensione $<5 \mathrm{~cm}^{2}$ sono state escluse dallo studio (Tabella 2).

I pazienti sono stati sottoposti ad un ciclo di tre trattamenti che sono stati effettuati ogni 30 giorni circa l'uno dall'altro, mentre solo in un paziente particolarmente critico è stato effettuato un quarto trattamento a distanza di circa due mesi dal terzo impianto.

Criteri di esclusione sono stati: insufficienza renale e scompenso cardiaco o epatico, patologie neoplastiche in atto, HIV e Epatite B o C, infezione sistemica in corso, anemizzazione severa o da malattie emopoietiche, quali la sindrome da disfunzione piastrinica e trombocitopenia.

\section{Concentrazione delle PBMNC}

Il sistema a filtrazione selettiva sterile e monouso utilizzato MonoCells (Athena Biomedical Innovation), ora commercializzato con il nome HemaTrate Blood Filtration System Cook Regentec, è un Dispositivo Medico marcato CE (classe IIB) che permette di processare 120 $\mathrm{mL}$ di sangue periferico in tempi brevi senza mai uscire dal campo operatorio, producendo un concentrato cellulare autologo di PBMNC con indicazione d'uso per terapia cellulare umana. Il concentrato cellulare ottenuto è stato estensivamente caratterizzato ${ }^{12}$ e produce una concentrazione media di cellule mononucleate di 200 milioni ogni $120 \mathrm{~mL}$ di sangue processato.

Tabella 1. Eziopatogenesi delle ulcere trattate.

\begin{tabular}{lc}
\hline Arteriose & 12 \\
\hline Miste & 3 \\
\hline Diabetiche & 6 \\
\hline Iatrogene & 1 \\
\hline
\end{tabular}

Tabella 2. Dimensioni delle ulcere trattate.

\begin{tabular}{lc}
$5-10 \mathrm{~cm}^{2}$ & 10 \\
\hline $10-20 \mathrm{~cm}^{2}$ & 5 \\
\hline $20-30 \mathrm{~cm}^{2}$ & 3 \\
\hline $30-50 \mathrm{~cm}^{2}$ & 1 \\
\hline $50-100 \mathrm{~cm}^{2}$ & 2 \\
$100-168 \mathrm{~cm}^{2}$ & 1
\end{tabular}


Brevemente, vengono prelevati $120 \mathrm{~mL}$ di sangue periferico dalle vene centrali dell'avambraccio anticoagulato con una soluzione di ACD al 10\%. Il prelievo viene caricato nella sacca superiore e per filtrazione per gravità processato (circa $10 \mathrm{~min}$ ). Le PBMNC vengono trattenute dal filtro e recuperate attraverso un contro-lavaggio con $10 \mathrm{ml}$ di soluzione fisiologica. Le cellule raccolte vengono immediatamente trasferite sterilmente in 10 siringhe da $1 \mathrm{cc}$ con ago da $21 \mathrm{G}$ e infiltrate.

\section{Impianto delle PBMNC}

L'impianto delle PBMNC è stato eseguito in sala operatoria (pazienti ricoverati in regime di ricovero ordinario o day hospital) sempre dopo debridement accurato.

L'impianto delle cellule è stato eseguito sotto guida ecografica, previa sedazione del paziente, con trattamento peri-vasale lungo il decorso delle arterie tibiali. Sono state infiltrate, per ogni sito di impianto circa $0,25 \mathrm{cc}$ di concentrato cellulare ottenuto ad $1 \mathrm{~cm}$ circa l'una dall'altra e ad $1 \mathrm{~cm}$ circa di profondità. Le cellule sono state impiantate anche nel tessuto peri-lesionale da trattare, infiltrando a circa $1 \mathrm{~cm}$ esternamente all'ulcera e con ago a $45^{\circ}$, in modo così da infiltrare il concentrato cellulare sotto l'ulcera stessa. Inoltre, $1 \mathrm{cc}$ di concentrato cellulare, è stata infiltrato all'interno del letto della ferita.

\section{Protocollo della gestione della lesione}

La medicazione finale è stata eseguita a piatto con garza non-adesiva e betadinata, quindi in tutti i casi è stato eseguito poi un bendaggio zincato e compressivo, con fascia coesiva leggera, a permanenza per almeno sette giorni successivi al trattamento. ${ }^{13}$

Le medicazioni hanno avuto una cadenza settimanale e sono state caratterizzate dal solo cambio delle garze a contatto con la lesione e il nuovo posizionamento del bendaggio all'arto inferiore trattato.

Il controllo della medicazione dopo sette giorni ha poi permesso di effettuare una valutazione oggettiva sulla riduzione del dolore caso per caso, oltre alla prevenzione di un'eventuale insorgenza di infezione locale.

Inoltre, ad ogni controllo, è stato eseguito un esame eco-color-doppler arterioso del tratto popliteo-tibiale all'arto inferiore trattato, per valutare l'insorgenza di una eventuale nuova rete di collaterali, collegati al circolo principale, lungo il decorso delle infiltrazioni eseguite in sala operatoria.

\section{Outcome clinico}

End point primario considerato è il tasso di guarigione completa della lesione. End points secondari sono stati invece la misurazione del dolore, tramite somministrazione di scala VAS, il tempo di guarigione della ferita stessa ed il tasso di recidiva
Il follow-up è stato eseguito a 1,3,4,6 e 12 mesi. Un secondo follow-up, su 5 pazienti, è stato eseguito a 4 anni.

\section{RISULTATI}

Delle 22 ulcere cutanee degli arti inferiori trattate, già alla fine della terza infiltrazione, cioè dopo il terzo mese di trattamento, sono state portate a guarigione 14 (circa il $64 \%$ ), mentre 8 lesioni (circa il 36\%), sono nettamente migliorate (Tabella 3 ).

Delle 8 ulcere migliorate con le tre procedure definite all'inizio dello studio, 3 sono giunte a guarigione spontaneamente dopo circa un mese dall'ultimo trattamento, 4 hanno necessitato, dopo circa un mese, di un innesto omologo di cute per poter arrivare a completa guarigione, che è avvenuta dopo circa sei mesi dall'inizio, mentre l'ultima, appartenente al gruppo delle arteriose, ha necessitato di un quarto trattamento, associato contemporaneamente ad innesto di cute omologa, ed è guarita circa dopo 12 mesi dall'inizio dei trattamenti. Il primo caso clinico riportato rappresenta un uomo affetto da ulcera arteriosa presente da circa 5 anni, che già dopo il primo trattamento ha potuto riposare a letto invece che in poltrona, mentre la guarigione finale è avvenuta tramite un innesto di cute omologa dopo il terzo ciclo di infiltrazioni (Appendice, Foto 1-4). Il secondo, invece, raffigura una donna affetta da ulcera mista, presente da circa 1 anno, che è giunta a guarigione spontaneamente dopo circa un mese dall'ultimo trattamento (Appendice, Foto 5-9), mentre il terzo rappresenta una ferita mista, con già compromissione linfatica, esitante da circa 6 mesi, che anch'essa è giunta a guarigione con innesto omologo (Appendice, Foto 10-11).

Invece per gli endpoints secondari, riguardante il tasso di recidive con follow-up a quattro anni delle lesioni trattate per gli otto pazienti portati a termine della procedura, non è stato più possibile rintracciare un paziente, due sono deceduti nel periodo selezionato, mentre gli altri cinque sono stati rivalutati dall'operatore stesso, che ha eseguito un ecocolordoppler arterioso dei vasi periferici. L'esame diagnostico ha dato un risultato molto importante, cioè in quattro pazienti sono stati ancora rilevati alcuni circoli collaterali formati alla fine dei trattamenti iniziali, mentre in quello affetto della lesione iatrogena non erano visibili, forse perché evidentemente non aveva alcuna patologia

Tabella 3. Risultati delle ulcere trattate al $3^{\circ}$ mese.

\begin{tabular}{lcc} 
& Guarite & Migliorate \\
Arteriose & 7 & 5 \\
\hline Miste & 1 & 2 \\
\hline Diabetiche & 5 & 1 \\
\hline Iatrogene & 1 & -
\end{tabular}


vascolare di base. I pazienti che sono stati rivalutati a quattro anni, erano portatori di 9 delle 22 ulcere iniziali, pari a circa il $41 \%$ di tutte le lesioni trattate inizialmente, erano tutti in buone condizioni di salute e facevano parte due del gruppo dismetabolico, uno rispettivamente dell'arterioso, del gruppo misto e appunto di quello iatrogeno. Nei quattro anni presi in considerazione, solo una paziente del gruppo dismetabolico ha dichiarato di aver ripresentato una lesione, ma distante da quella inizialmente trattata, per un diabete transitoriamente non più compensato e già guarita in altra sede, mentre nessuno di tutti gli altri era stato affetto da una nuova ulcera nel periodo selezionato (Tabella 4). I casi clinici presentati come follow-up a 4 anni hanno voluto comprendere tre pazienti di due gruppi differenti, rispettivamente uno dell'arterioso (Appendice, Foto 12-14) e due del diabetico (Appendice, Foto 15-20), di cui il primo di quest'ultimo gruppo è stato appunto quello che ha dovuto subire una quarta infiltrazione prima di giungere alla guarigione completa.

Eseguendo un'analisi più dettagliata degli end points secondari, è stato rilevato, con una Scala Visuo-Analogica (VAS) valutante il dolore prima e dopo i trattamenti, che tutti i pazienti, già dopo sette giorni dall'inizio del trattamento hanno avuto una riduzione della sintomatologia valutata come dolore notturno riferito, mentre si era ridotto dopo i tre cicli per più dei $2 / 3$ del valore iniziale (Figura 1) Per quanto riguarda invece i tempi di guarigione, abbiamo avuto sicuramente un'accelerazione del processo visto che 14 ulcere sono guarite già al terzo mese di trattamento, cioè alla fine dell'ultimo ciclo, mentre delle altre 8,3 sono guarite entro un mese, 4 dopo tre e solo una dopo più sei mesi dalla fine del ciclo di trattamenti stabiliti (Figura 2).

In tutti i pazienti trattati, il chirurgo-operatore che ha eseguito l'ecocolordoppler lungo il decorso delle infiltrazioni, ha notato un costante incremento di neo-vasi sottocutanei rilevabili con la funzione power-doppler e color-doppler dell'ecografo, per cui è stato sottointeso un aumento del flusso nelle aree considerate ipovascolarizzate all'inizio del trattamento.

Non si sono registrati effetti collaterali o reazioni avverse ne maggiori e ne minori in nessun paziente trattato con PBMNC autologhe.

Tabella 4. Eziologia delle ulcere con follow-up a 4 anni.

\begin{tabular}{lcc} 
& Guarite & Recidive \\
Arteriose & 2 & - \\
\hline Miste & 2 & - \\
\hline Diabetiche & 4 & 1 \\
\hline Iatrogene & 1 & -
\end{tabular}

\section{DISCUSSIONE}

L'ulcera, per sua natura, non tende spontaneamente alla guarigione, ma con la correzione delle condizioni patologiche, che spesso stanno alla base della sua formazione, e con l'utilizzo di terapie appropriate, la lesione tende a ripararsi ed a essere colmata da un tessuto connettivo epiteliale, di aspetto differente alla cute sana circostante.

Dalle molteplici osservazioni cliniche e dai risultati diagnostici sembra di poter dedurre che tre fattori ricorrono costantemente nella patogenesi delle ulcere croniche degli arti inferiori: il ridotto afflusso o deflusso di sangue e/o la presenza di una patologia sistemica in atto. La presenza di uno di questi fattori, o la loro combinazione, è senza dubbio indispensabile per l'origine delle ulcere croniche degli arti inferiori. ${ }^{13}$

Il ruolo fondamentale dei monociti-macrofagi nella guarigione delle lesioni è stato descritto oramai in numerosi lavori. ${ }^{6,14-16}$ In particolare diversi studi ${ }^{17,18}$ indicano i monociti-macrofagi come un nuovo ed interessante target terapeutico sul quale agire per riattivare la guarigione

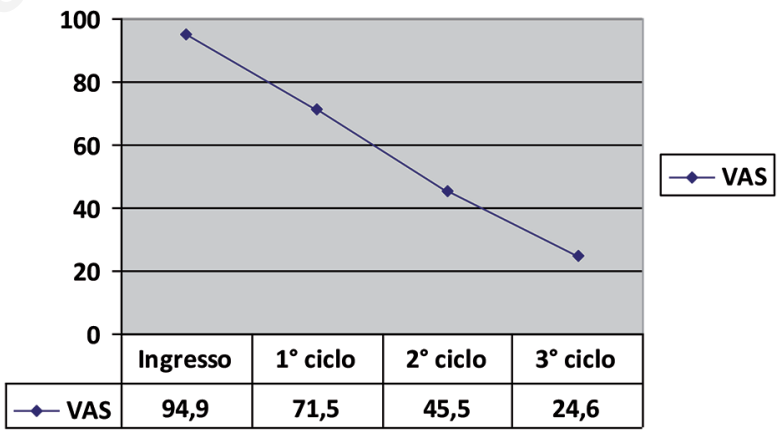

Figura 1. Il grafico rappresenta i valori della Scala VAS ad ogni ciclo.

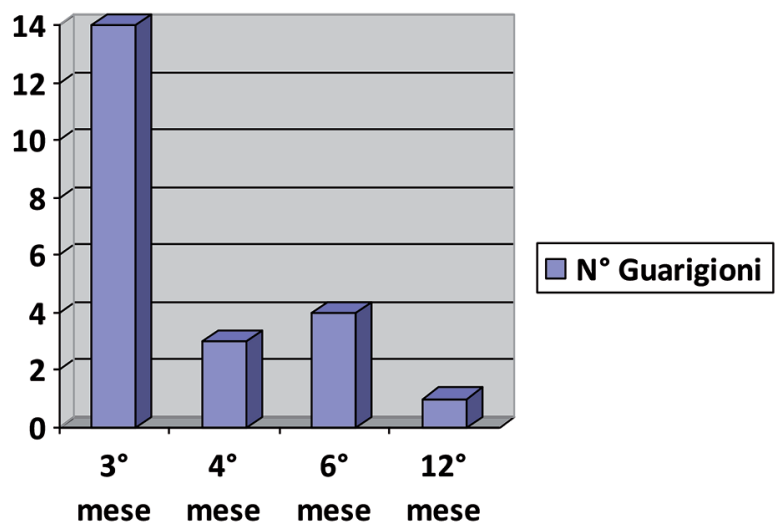

Figura 2. Il grafico rappresenta il numero delle ulcere guarite a 12 mesi. 
delle lesioni croniche e lo stesso concetto viene approfondito nel suo aspetto cellulare e molecolare. ${ }^{19-21}$

È stato inoltre dimostrato che i macrofagi controllano la trascrizione del collagene durante le prime fasi del wound healing e che i fibroblasti del derma, stimolati dai fattori paracrini rilasciati dai macrofagi, attivati nella forma anti-infiammatoria e rigenerativa detta M2, mostrano un aumentato indice di proliferazione. ${ }^{16,22}$

Evidenze in vitro ed in vivo indicano un ruolo fondamentale dei monociti-macrofagi nei processi di rigenerazione tessutale, infatti mostrano come questi tipi di cellule siano in grado di creare lo spazio nella matrice extracellulare, favorire la diffusione di cellule progenitrici (comprese quelle vascolari), attivando la co-migrazione, e di cooperare con le cellule progenitrici residenti, formando quindi un nuovo tessuto vascolarizzato. ${ }^{8,23}$

I monociti sono presenti nei tessuti umani come cellule circolanti nel sangue periferico e come cellule residenti in tutti i tessuti nella forma di macrofagi, che sono cellule ad alta plasticità capaci di interagire con molteplici popolazioni cellulari e cellule staminali residenti nei tessuti. ${ }^{19,24}$ Inoltre, i monociti secernono interleuchina-1, che facilita l'attivazione dei linfociti $\mathrm{T}$ da parte dei monociti-macrofagi, promuovendo la moltiplicazione dei fibroblasti e quindi, di conseguenza, la guarigione delle ferite. ${ }^{25,26}$

I macrofagi residenti nei tessuti sono in forma quiescente detta $\mathrm{M} \varphi$, ma vengono attivati dai batteri o da citochine infiammatorie nella forma M1, nelle lesioni che guariscono la forma M1 viene convertita nella forma rigenerativa $\mathrm{M} 2$ che provoca il remodelling della lesione e la sua guarigione. Nelle lesioni croniche, al contrario, questa conversione, detta polarizzazione, non avviene e la lesione permane in uno stato infiammatorio che ne impedisce la guarigione ${ }^{15}$ quali le lesioni cutanee croniche ed in particolare il piede diabetico. ${ }^{27} \mathrm{E}$ stato dimostrato che impiantando un concentrato cellulare di monociti in un tessuto infiammato, i macrofagi M1 vengono "polarizzati" nella forma M2, con la partenza della fase rigenerativa. ${ }^{28,29}$ Non è chiaro se il meccanismo di questo switch comprende il coinvolgimento di precursori circolanti o la rieducazione di cellule in situ, ma è stato dimostrato che alcune sottopopolazioni specializzate dei linfociti $\mathrm{T}$ (Th1, Th2, Tregs) sono in grado di attivare la polarizzazione dei macrofagi ed esibiscono inoltre una flessibilità e plasticità mai osservata prima. ${ }^{30}$

Il termine angiogenesi terapeutica è stato proposto la prima volta nel 1993 da Hockel per descrivere interventi diretti ad indurre la crescita di vasi sanguigni in aree di ipovascolarizzazione ed è noto da tempo che nel paziente con patologie ostruttive delle arterie, fisiologicamente, si verificano due diverse forme di vascolarizzazione compensatorie, l'angiogenesi e l'arteriogenesi.

Per angiogenesi si intende lo sviluppo di un nuovo circolo capillare per gemmazione (sprouting) a partire da capillari pre-esistenti, in risposta all'ipossia tessutale locale ed è mediato dal rilascio di citochine (VEGF e altre citochine). I capillari che ne derivano sono molto piccoli, con un diametro di circa 10-20 um, che da soli non riescono a compensare la mancanza di flusso sanguigno dovuta all'occlusione arteriosa (legge di Hagen Poiseuille).

L'arteriogenesi, al contrario, è la trasformazione di arteriole pre-esistenti in arterie collaterali funzionali, in grado di compensare la riduzione di flusso. Il diametro originale di una piccola arteriola può aumentare anche di 20 volte durante il processo di arteriogenesi e questo viene innescato da un aumento dello shear stress sull'endotelio che provoca, a sua volta, un aumento delle molecole di adesione (MCP-1) per i monociti circolanti che si accumulano attorno alle arterie in stato proliferativo e provvedono al rilascio di citochine e fattori di crescita, indicando così che i monociti-macrofagi giocano un ruolo fondamentale in questo processo. ${ }^{31,32} \mathrm{E}$ ' stato anche riportato, oltre all'aumento dei monociti-macrofagi, anche un aumento di linfociti $\mathrm{T}$ attorno alle nuove collaterali, indicando quindi un importanza fondamentale anche di questo tipo di cellule nella crescita dei nuovi vasi..$^{25,33-35}$

Nonostante questo, il meccanismo fisiologico di compensazione di arteriogenesi attraverso il reclutamento dei monociti non funziona in un grande numero di pazienti, in quanto la ridotta perfusione non permette ai monociti e ai linfociti di arrivare nel tessuto danneggiato in quantità sufficiente. Particolarmente interessante la correlazione dimostrata in numerosi studi tra fattori di rischio dell'ischemia critica e basso numero di EPC-monociti circolanti; particolarmente questo meccanismo è compromesso nei pazienti diabetici, fumatori e con iper-lipidemia, nei quali la concentrazione delle EPC-monocitaria misurata risulta fortemente diminuita. ${ }^{36,37}$

I risultati osservati sulle lesioni croniche trattate con PBMNC nel nostro centro possono essere correlati sia alla capacità angiogenica ed arteriogenica delle $\mathrm{PBMNC}$, sia alla capacità di polarizzazione in $\mathrm{M} 2$ in grado di cambiare lo stato infiammatorio del tessuto.

I dati osservati sono in linea con quanto precedentemente osservato sia nel piede diabetico ${ }^{10,38,39}$ sia in lesioni croniche con altra eziologia ${ }^{40,41}$ in studi che hanno utilizzato le PBMNC prodotte con sistema a filtrazione selettiva. Questa tecnologa innovativa rappresenta una tecnologia avanzata nell'isolamento e concentrazione delle cellule PBMNC, apportando diversi importanti vantaggi rispetto alle tecnologie basate sulla centrifugazione, preservando sia l'integrità di membrana, ma soprattutto il rilascio delle molecole attive sul quale si basa il meccanismo d'azione biologico (effetto paracrino). Inoltre la facilità e la velocità d'uso, l'alta riproducibilità (la metodica non è operatore dipendente) la rendono estremamente maneggevole. 
Il dato originale delle nostre osservazioni è che nei cinque pazienti che sono stati sottoposti a follow-up a 4 anni, i circoli collaterali erano ancora rilevabili e nessuno dei pazienti aveva mostrato una recidiva nell'arto trattato.

Questi dati sono chiaramente semplici case reports di pazienti con ulcere ad eziologia mista, sebbene tutte nonhealing a 6 mesi: nessuna considerazione statistica è chiaramente possibile su un numero di casi estremamente limitato e riferiti ad un gruppo non omogeneo per definizione.

\section{CONCLUSIONI}

Le ulcere croniche degli arti inferiori, data la loro notevole frequenza ed invalidità prolungata, è una patologia oramai considerata sociale, per cui richiede da parte dei medici e dei pazienti stessi la massima attenzione che, in pratica, consiste nella prevenzione scrupolosamente condotta nel tempo, in modo da limitarne l'evoluzione e la comparsa di complicanze locali e sistemiche.

Sia il superamento del concetto di malattia come espressione di processo localizzato, che il notevole approfondimento conoscitivo degli ultimi anni, hanno concorso nel far inquadrare le ulcere cutanee degli arti inferiori come espressione di una patologia d'organismo e non più come un'entità a sé stante.

La terapia delle ulcere cutanee è abbastanza complessa, in considerazione della molteplicità dei meccanismi che ne determinano la comparsa e ne alimentano la cronicizzazione.

Inoltre esistono dei fattori esterni alla lesione che influenzano la guarigione delle stesse, tra cui, la principale, l'età del paziente ed il suo stato generale, infatti nella nostra esperienza la totalità dei casi è over- 70 , quindi con alte percentuali di comorbilità.

La terapia cellulare autologa con PBMNC presenta un profilo di sicurezza favorevole con un tasso di eventi avversi molto basso e nessun aumento di eventi gravi, in linea con quanto riportato da numerose meta-analisi, ${ }^{42-44}$ e ha un ottimo rapporto positivo rischio-beneficio, per cui può essere una valida opzione terapeutica, in particolare per quei pazienti non responder ai trattamenti standard.

Quindi il mancato rilievo di effetti collaterali, la bassa invasività e la ripetibilità, permettono di considerare ottima la tollerabilità della terapia cellulare con PBMNC nel trattamento delle ulcere degli arti inferiori. Questa caratteristica è di estrema importanza per una modalità terapeutica che è destinata ad essere utilizzata da una popolazione prevalentemente anziana come quella vulnologica. Del resto, molti dei pazienti trattati nello studio erano portatori di altre patologie concomitanti, eppure hanno dimostrato di ben tollerare l'eventuale stress portato da tale metodica terapeutica.

In conclusione, questi otto case reports sul trattamenti con PBMNC autologhe di ulcere a diversa eziologia, sug- geriscono l'efficacia e l'ottima tollerabilità nel trattamento delle ulcere degli arti inferiori, con un notevole miglioramento della qualità della vita dei pazienti sottoposti a tale terapia.

\section{BIBLIOGRAFIA}

1. Agus GB, Allegra C, Arpaia G, et al. Linee guida. Collegio Italiano di Flebologia - Revisione 2013. Acta Phlebologica 2013;14.

2. Platsidaki E, Kouris A, Christodoulou C. Psychosocial aspects in patients with chronic leg ulcers. Wounds 2017;29:306-10.

3. Patel AS, Smith A, Nucera S, et al. TIE2-expressing monocytes/macrophages regulate revascularization of the ischemic limb. EMBO Mol Med 2013;5:858-69.

4. Krishnasamy K, Limbourg A, Kapanadze T, et al. Blood vessel control of macrophage maturation promotes arteriogenesis in ischemia. Nat Commun 2017;8:952.

5. Van Weel V, Toes REM, Seghers L, et al. Natural killer cells and CD4+ T-cells modulate collateral artery development. Arterioscler Thromb Vasc Biol 2007;27:2310-8.

6. Wynn TA, Vannella KM. Macrophages in tissue repair, regeneration, and fibrosis. Immunity 2016;44:450-62.

7. Larouche J, Sheoran S, Maruyama K, Martino MM. Immune regulation of skin wound healing: mechanisms and novel therapeutic targets. Adv Wound Care 2018;7:209-31.

8. Gurevich DB, Severn CE, Twomey C, et al. Live imaging of wound angiogenesis reveals macrophage orchestrated vessel sprouting and regression. EMBO J 2018;37:e97786.

9. De Angelis B, Gentile P, Orlandi F, et al. Limb rescue: a new autologous-peripheral blood mononuclear cells technology in critical limb ischemia and chronic ulcers. Tissue Eng Part C Methods 2015;21:423-35.

10. Persiani F, Paolini A, Camilli D, et al. Peripheral blood mononuclear cells therapy for treatment of lower limb ischemia in diabetic patients: a single-center experience. Ann Vasc Surg 2018;53:190-6.

11. Rigato M, Monami M, Fadini GP. Autologous cell therapy for peripheral arterial disease: systematic review and metaanalysis of randomized, nonrandomized, and noncontrolled studies. Circ Res 2017;120:1326-40.

12. Spaltro G, Straino S, Gambini E, et al. Characterization of the pall celeris system as a point-of-care device for therapeutic angiogenesis. Cytotherapy 2015;17:1302-13.

13. Mariani F, Macciò A, et al. Consensus conference on compression therapy. Airali; 2018: pp. 44-9.

14. Ogle ME, Segar CE, Sridhar S, Botchwey EA. Monocytes and macrophages in tissue repair: Implications for immunoregenerative biomaterial design. Exp Biol Med 2016;241:1084-97.

15. Krzyszczyk P, Schloss R, Palmer A, Berthiaume F. The role of macrophages in acute and chronic wound healing and interventions to promote pro-wound healing phenotypes. Front Physiol 2018;9:419.

16. Rodero MP, Legrand JMD, Bou-Gharios G, Khosrotehrani $\mathrm{K}$. Wound-associated macrophages control collagen $1 \alpha 2$ transcription during the early stages of skin wound healing. Exp Dermatol 2013;22:143-5. 
17. Do N, Willenborg S, Eming SA. Molecular mechanisms and functional consequences of blood monocyte recruitment and macrophage activation in tissue repair and fibrosis. Exp Dermatol 2015;24:20-2.

18. Willenborg S, Eming SA. Macrophages-sensors and effectors coordinating skin damage and repair. J German Soc Dermatol 2014;12:214-21.

19. Moore EM, Maestas, Jr. DR, Comeau HY, Elisseeff JH. The immune system and its contribution to variability in regenerative medicine. Tissue Eng Part B Rev 2020;ten.TEB.2019.0335.

20. Julier Z, Park AJ, Briquez PS, et al. Promoting tissue regeneration by modulating the immune system. Acta Biomater 2017;53:13-28.

21. Forbes SJ, Rosenthal N. Preparing the ground for tissue regeneration: From mechanism to therapy. Nat Med 2014;20:857-69.

22. Ta Ploeger D, Hosper NA, Schipper M, et al. Cell plasticity in wound healing: paracrine factors of M1/ M2 polarized macrophages influence the phenotypical state of dermal fibroblasts. Cell Comm Signaling 2013;11:29.

23. Anghelina M, Krishnan P, Moldovan L, Moldovan NI. Monocytes/macrophages cooperate with progenitor cells during neovascularization and tissue repair: Conversion of cell columns into fibrovascular bundles. Am J Pathol 2006; 168:529-41.

24. Naik S, Larsen SB, Cowley CJ, Fuchs E. Leading edge two to tango: dialog between immunity and stem cells in health and disease. Cell 2018;175:908-20.

25. Sharma A, Rudra D. Emerging functions of regulatory t cells in tissue homeostasis. Front Immunol 2018;9:883.

26. Li J, Tan J, Martino MM, Lui KO. Regulatory T-cells: Potential regulator of tissue repair and regeneration. Front Immunol 2018;9:585.

27. Torres-Castro I, Arroyo-Camarena ÚD, Martínez-Reyes CP, et al. Human monocytes and macrophages undergo M1-type inflammatory polarization in response to high levels of glucose. Immunol Lett 2016;176:81-9.

28. Di Pardo A, Cappello E, Pepe G, et al. Infusion of autologous-peripheral blood mononuclear cells : a new approach for limb salvage in patients with diabetes. In: 7th International Diabetic Foot Congress abu Dhabi. IFD Congress Abu Dhabi 4-8 December 2017.

29. Arnold L, Henry A, Poron F, et al. Infl ammatory monocytes recruited after skeletal muscle injury switch into antiinfl ammatory macrophages to support myogenesis. J Exp Med 2007;204:1057-69.

30. Biswas SK, Mantovani A. Macrophage plasticity and interaction with lymphocyte subsets: Cancer as a paradigm. Nat Immunol 2010;11:889-96.
31. Murray PJ, Wynn TA. Protective and pathogenic functions of macrophage subsets. Nat Rev Immunol 2011;11:723-737.

32. Kimball A, Schaller M, Joshi A, et al. Ly6C Hi blood monocyte/macrophage drive chronic inflammation and impair wound healing in diabetes mellitus. Arterioscler Thromb Vasc Biol 2018;38:1102-14.

33. Liang C, Yang KY, Chan VW, et al. CD8+ T-cell plasticity regulates vascular regeneration in type-2 diabetes. Theranostics 2020;10:4217-32.

34. Kwee BJ, Seo BR, Najibi AJ, et al. Treating ischemia via recruitment of antigen-specific $\mathrm{T}$ cells. Sci Adv 2019; 5: eaav6313.

35. Leung OM, Li J, Li X, et al. Regulatory T cells promote apelin-mediated sprouting angiogenesis in type 2 diabetes. Cell Rep 2018;24:1610-26.

36. Churdchomjan W, Kheolamai P, Manochantr S, et al. Comparison of endothelial progenitor cell function in type 2 diabetes with good and poor glycemic control. BMC Endocr Disord 2010;10:5.

37. Dopheide JF, Geissler P, Rubrech J, et al. Inflammation is associated with a reduced number of pro-angiogenic Tie-2 monocytes and endothelial progenitor cells in patients with critical limb ischemia. Angiogenesis 2016;19:67-78.

38. Caravaggi CMF, Panunzi Andrea, Sangalli E SG. Clinical outcome of autologous PB-MNC in treatment of non infected ischemic DFU (3C TUC) in non option CLI. In: 6th World Union of Wound Healing Societes; 2020.

39. Scatena A, Maioli F PP, Ventoruzzo G LF, et al. Does autologous peripheral mononuclear cells implant allow foot surgery in diabetic patients with critical limb ischaemia not eligible for revascularization? In: 8th International Symposium Diabetic Foot. The Hague- Netherlands; 2019: p. 95.

40. Colonna MR, Flavia L, Gabriele D, et al. Regenerative approaches regenerative in wound healing: new new alternatives for older tools. In: Alexandrescu V (ed). Wound Healing- New Insight into Ancient Challenges. IntechOpen; 2016: pp. 155-63.

41. Palermo C, Sanfiorenzo A, Trigona C, Bernardini G. Role of monocytes in the treatment of chronic limb ischemia and "hard to heal" ulcers. J Vasc Surg 2018;68:e119.

42. Benoit E, O'donnell TF, Patel AN. Safety and efficacy of autologous cell therapy in critical limb ischemia: a systematic review. Cell Transplant 2013;22:545-62.

43. Gao W, Chen D, Liu G, Ran X. Autologous stem cell therapy for peripheral arterial disease: a systematic review and metaanalysis of randomized controlled trials. Stem Cell Res Ther 2019;10:140

44. Dubský M, Jirkovská A, Bem R, et al. Cell therapy of critical limb ischemia in diabetic patients - State of art. Diabetes Res Clin Pract 2017;126:263-71. 